OPEN ACCESS

Edited by: Michel Chérel, University of Nantes, France

Reviewed by: Sandrine Huclier, University of Nantes, France Patrice Hildgen, Université de Montréal, Canada

*Correspondence: Nicolas Lepareur, Centre Eugene Marquis, Avenue de la Bataille Flandres-Dunkerque, CS 44229, Rennes Cedex 35042, France n.lepareur@rennes.unicancer.fr

tPresent address: Maëva Bocqué, CNRS UMR 5223, Laboratory of Polymer Materials Engineering, University Claude Bernard Lyon 1, Lyon, France

Specialty section: This article was submitted to Nuclear Medicine, a section of the journal

Frontiers in Medicine

Received: 02 June 2015 Accepted: 21 August 2015 Published: 04 September 2015

Citation:

Lepareur N, Leal E Costa L, Bocqué M, Blondelle C, Ruello C, Desjulets M, Noiret $N$ and CammasMarion S (2015) Development of

biocompatible and functional polymeric nanoparticles for site-specific delivery of radionuclides.

Front. Med. 2:63.

doi: 10.3389/fmed.2015.00063

\section{Development of biocompatible and functional polymeric nanoparticles for site-specific delivery of radionuclides}

\author{
Nicolas Lepareur 1,2*, Loleh Leal E Costa ${ }^{1,3}$, Maëva Bocqué ${ }^{1,3 \dagger}$, Clément Blondelle ${ }^{1,3}$, \\ Clément Ruello ${ }^{1,3}$, Marie Desjulets ${ }^{1}$, Nicolas Noiret ${ }^{3,4}$ and Sandrine Cammas-Marion ${ }^{3,4}$ \\ ${ }^{1}$ Comprehensive Cancer Centre Eugene Marquis, Rennes, France, ${ }^{2}$ INSERM UMR-S 991, Rennes, France, ${ }^{3}$ Ecole Nationale \\ Supérieure de Chimie de Rennes (ENSCR), Rennes, France, ${ }^{4}$ UMR 6226 CNRS, Institute of Chemical Sciences of Rennes, \\ Rennes, France
}

Introduction: Encapsulation of biologically active molecules into nanoparticles (NPs), for site-specific delivery, is a fast growing area. These NPs must be biocompatible, non-toxic, and able to release their load in a controlled way. We have developed a series of NPs based on (bio)degradable and biocompatible poly(malic acid) derivatives, poly(benzyl malate) (PMLABe), with its PEG-grafted stealth analog and target-specific biotin-PEG- $b$ $\mathrm{PMLABe}$ one. A lipophilic radiotracer has then been encapsulated into these NPs.

Methods: Monomers were synthesized from DL-aspartic acid. $\mathrm{PEG}_{42}-b-\mathrm{PMLABe}_{73}$ and Biot-PEG $\mathrm{G}_{66}-b-\mathrm{PMLABe}_{73}$ block copolymers were obtained by anionic ring-opening polymerization of benzyl malolactonate in presence of $\alpha$-methoxy- $\omega$-carboxy-PEG $\mathrm{G}_{42}$ and $\alpha$-biotin- $\omega$-carboxy-PEG ${ }_{66}$ as initiators. NPs were prepared by nanoprecipitation. Size, polydispersity, and zeta potential were measured by dynamic light scattering (DLS) and zetametry. ${ }^{99 m}$ Tc-SSS was prepared as previously described. Encapsulation efficacy was assessed by varying different parameters, such as encapsulation with preformed NPs or during their formation, influence of the solvent, and of the method to prepare the

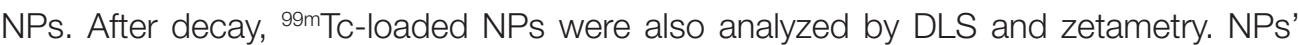
morphology was assessed by transmission electron microscopy.

Results: ${ }^{99 m T C-S S S}$ was added during nanoprecipitation, using two different methods, to ensure good encapsulation. Radiolabeled NPs present increased diameters, with identical low polydispersity indexes and negative zeta potentials in comparison to non-radiolabeled NPs.

Conclusion: A radiotracer was successfully encapsulated, but some further optimization is still needed. The next step will be to modify these radiolabeled NPs with a hepatotrope peptide, and to replace ${ }^{99 \mathrm{~m}} \mathrm{Tc}$ with ${ }^{188} \mathrm{Re}$ for therapy. Our team is also working on drugs' encapsulation and grafting of a fluorescent probe. Combining these modalities is of interest for combined chemo-/radiotherapy, bimodal imaging, and/or theranostic approach.

Keywords: functional nanoparticles, site-specific targeting, degradable poly(benzyl malate) derivatives, radionuclide encapsulation, technetium-99m 


\section{Introduction}

Nanomedicine, the use of nanoparticles (NPs) in medicine, is an ever-growing research field as illustrated by the huge amount of scientific publications and the important number of formulations registered for clinical trials, especially for cancer therapies (1). Such a growing interest results from the urgent need for highly efficient anti-cancer drug delivery systems allowing an increased efficacy of the drugs together with a decrease of their non-specific distribution and toxicity (2-4). Indeed, despite better knowledge and understanding of cancer biology, significant improvements in cancer treatments are still needed, specifically to address a large amount of drugs at the site of action, to decrease their toxic side effects and to simplify the administration protocols. In this context, nanoparticulate systems (liposomes, micelles, polymerbased NPs, etc.) have attracted the interest of many researchers and numerous systems have been developed (1). However, the prepared NPs have to answer to very strict specifications: (i) they have to be non-toxic and biocompatible at any stage of their life, until their complete excretion from the body; (ii) they need to have stealth properties and reduced immunogenicity together with ability to carry large amount of drug and to deliver it at a specific site in a sustainable way. Even if undeniable progresses have been achieved in the design of the ideal drug carriers as evidenced by the Food and Drug Administration (FDA) approval obtained by some nanovectors and by the number of NPs in preclinical or clinical phases $(1,5,6)$, several properties, such as biocompatibility, drug loading capacity and site-specificity of drug release, need to be further improved. Among the nanoplatforms designed to address these objectives, polymer-based NPs have attracted much interest mainly because of the versatile characteristics of polymers allowing adjustment of the physico-chemical and biological properties of the corresponding nanocarriers. Consequently, several families of polymers have been developed to formulate multifunctional drug carriers with properties adjusted to the considered application (2). However, when designing a polymer family, one must keep in mind that the resulting materials are prepared to be used in vivo and that they have to respect the very strict specifications defined above. Another property which can be of importance is the (bio)degradability of the polymers constituting the drug delivery systems, allowing their elimination thanks to the (bio) degradation of the copolymers constituting the NPs, thus avoiding NPs accumulation in the body. However, this means that the degradation process must lead to non-immunogenic, biocompatible, and/or (bio)assimilable low molecular weight molecules that can be eliminated from the body. In this context, several (bio) degradable polymers have been developed and formulated under various nanoplatforms with more or less success $(7,8)$.

Therefore, the design of (bio)degradable polymers, whose physico-chemical and biological properties can be adjusted either by copolymerization, chemical modifications, or both, is of great importance $(9,10)$. Poly $(\beta$-malic acid) (PMLA) is a very good candidate due to the presence of carboxylic acid pendant groups, which can be chemically modified to introduce molecules of interest, and ester cleavable bounds within the polymer backbone, allowing the degradation of the polymer main chain into biocompatible metabolites (11-13).
Recently, we have synthesized and characterized two amphiphilic derivatives of PMLA presenting stealth properties, the poly(ethylene glycol)-b-poly(benzyl malate) ( $\mathrm{PEG}_{42}-b$ $\mathrm{PMLABe}_{73}$ ), and targeting properties, the biotin- $\mathrm{PEG}_{66}-b-\mathrm{PM}-$ $\mathrm{LABe}_{73}$. These block copolymers are able to self-assemble in aqueous media, leading to well-defined NPs designed for sitespecific sustainable drug release in the frame of hepatocellular carcinoma (HCC). We showed that an anti-cancer drug model, the Doxorubicin (Dox), can be encapsulated into both stealth and targeted NPs with quite high encapsulation efficiency $(14,15)$ and that both kind of empty NPs have no significant toxicity against normal and cancer cell lines even at high concentrations (14-16).

Among molecules of interest to be encapsulated, one can include radioisotopes, either for imaging (diagnostic) or therapy, or even both (theranostics). There is an abundant, and ever-growing literature on the subject (17-23). All types of NPs have been radiolabeled (24), from micelles (25), polymeric NPs (26), liposomes (27), nanocapsules (28), or dendrimers (29), to inorganic ones (30). Most of the applications have been in cancer imaging and/or therapy (17), but some applications in cardiovascular $(20,23)$ and inflammation/infection $(31,32)$ can be found. Radiolabeling NPs enables the following of the NPs in vivo (33), particularly as an image-guided system for targeted delivery $(34,35)$, or for the controlled delivery of a therapeutic radionuclide (36).

Due to its favorable physical and chemical properties, such as its 6-h half-life, its low energy gamma emission of $140 \mathrm{keV}$, and relatively low isotope cost, generator-produced technetium- $99 \mathrm{~m}$ $\left({ }^{99 \mathrm{~m}} \mathrm{Tc}\right)$ has been the most used radionuclide in routine nuclear medicine and is still the subject of numerous investigations, in the search for new radiopharmaceuticals. Logically, attempts to label NPs with ${ }^{99 \mathrm{~m}} \mathrm{Tc}$ have been done $(26,30,37) .{ }^{99 \mathrm{~m}} \mathrm{Tc}$ has also the advantage of being part of a matched pair with rhenium-186/188, two isotopes that could be used as therapeutic radionuclides. Rhenium-188 $\left({ }^{188} \mathrm{Re}\right)$, which is also conveniently generatorproduced, like ${ }^{99 \mathrm{~m}} \mathrm{Tc}$, has great potential for radionuclide therapy $(38,39)$. Moreover, due to similar chemistries, it can be easily replaced with rhenium-188 $\left(\mathrm{E}_{\beta \max }=2.1 \mathrm{MeV}, \mathrm{E}_{\gamma}=155 \mathrm{keV}(15 \%)\right.$, $\mathrm{t}_{1 / 2}=17 \mathrm{~h}$ ) for therapy purposes $(40-42)$.

Because of the growing interest in the site-specific and controlled delivery of radionuclides, we investigated the possibilities to entrap a lipophilic radiotracer, ${ }^{99 \mathrm{~m}} \mathrm{Tc}$-SSS, inside NPs based on $\mathrm{PEG}_{42}-b-\mathrm{PMLABe}_{73}$ or Biot-PEG 6 - $b-\mathrm{PMLABe}_{73}$. Several methods were used to encapsulate ${ }^{99 \mathrm{~m}} \mathrm{Tc}-\mathrm{SSS}$ with more or less success. The corresponding radiolabeled NPs were characterized in terms of encapsulation efficiency, diameters, polydispersities, zeta potentials, and morphology.

\section{Materials and Methods}

\section{Materials}

Nuclear magnetic resonance spectra ( ${ }^{1} \mathrm{H}$ NMR) were recorded at $400 \mathrm{MHz}$ on a Brucker ARX400 instrument (Billerica, MA, USA). Data are reported as follows: chemical shift (multiplicity, number of hydrogen). The chemical shifts $(\delta)$ are reported as parts per million (ppm) referenced to the appropriate residual solvent peak. Abbreviations are as follows: $s$ (singlet), 
$d$ (doublet), $t$ (triplet), $q$ (quartet), dd (doublet of doublet), $m$ (multiplet).

Weight average molecular weight $(\mathrm{Mw})$ and molecular weight distribution $(\mathrm{Mw} / \mathrm{Mn}=\mathrm{Ip})$ values were determined by size exclusion chromatography (SEC) in THF at $40^{\circ} \mathrm{C}$ (flow rate $=1.0 \mathrm{~mL} \cdot \mathrm{min}^{-1}$ ) on a GPC 2502 Viscotek apparatus (Malvern Instruments Ltd, Malvern, UK) equipped with a refractive index detector, a LT5000L mixed medium org $300 \mathrm{~mm} \times 7.8 \mathrm{~mm}$ gel column and a GPC/SEC OmniSEC software. The polymer samples were dissolved in THF $\left(2 \mathrm{mg} \cdot \mathrm{mL}^{-1}\right)$. All elution curves were calibrated with poly(styrene) standards.

The dynamic light scattering (DLS) measurements were recorded on a Delsa ${ }^{\mathrm{TM}}$ Nano Beckman Coulter apparatus (Fullerton, CA, USA) at $25^{\circ} \mathrm{C}$ in water. The obtained correlation curve allows calculating the diffusion coefficient $(D)$ using the CONTIN method. The hydrodynamic radius ( $\mathrm{Rh}$ ), and therefore the hydrodynamic diameter (Dh), is then calculated from this diffusion coefficient $D$ using the Stokes-Einstein equation where $\mathrm{k}$ is the Boltzmann constant, $T$ the temperature, and $\eta$ the medium viscosity (fixed at $0.8878 \mathrm{cP}$ ): $D=\mathrm{kT} / 6 \pi \eta \mathrm{Rh}$.

The zeta potential was measured by zetametry using a Delsa ${ }^{\mathrm{TM}}$ Nano Beckman Coulter apparatus (Fullerton, CA, USA) at $25^{\circ} \mathrm{C}$ in water. Laser Doppler electrophoresis in a phase mode was conducted with sequential fast and slow field reversal applying a potential of $\pm 150 \mathrm{~V}$. The measured electrophoretic mobility $(\mu)$ was then converted to zeta potential using the Smoluchowski approximation.

Transmission electron microscopy (TEM) was realized using a Jeol 2100 LAB6 microscope (Peabody, MA, USA) equipped with a Gatan Orius 200D camera (Pleasanton, CA, USA). UV spectra were recorded on a Secomam apparatus (Alès, France) at $485 \mathrm{~nm}$. Activity measurements were done in a CRC-127R well-counter (Capintec Inc., Ramsey NJ, USA).

Radiochemical purity (RCP) of the complex was determined using a Perkin-Elmer Cyclone Storage Phosphor Imager (Waltham, MA, USA), with the Packard Optiquant v05.00 software. All chemicals were used as received. Anhydrous THF was obtained by distillation over sodium/benzophenone under $\mathrm{N}_{2}$ atmosphere.

\section{Synthesis of the Monomers and Polymers}

The monomer, benzyl malolactonate (MLABe), was synthesized from DL-aspartic acid according to the previously reported synthesis (43). $\mathrm{PEG}_{42}-b$-PMLABe $\mathrm{PM}_{73}$ and Biot-PEG 6 - $b$-PMLABe $\mathrm{PM}_{73}$ block copolymers were obtained by anionic ring opening polymerization of MLABe in presence of, respectively, $\alpha$-methoxy $\omega$-carboxy poly(ethylene glycol), $\mathrm{PEG}_{42}-\mathrm{CO}_{2} \mathrm{H}$, and $\alpha$-biotin $\omega$-carboxy poly(ethylene glycol), Biot- $\mathrm{PEG}_{66}-\mathrm{CO}_{2} \mathrm{H}$, as initiators following a protocol described elsewhere (14).

\section{PEG $_{42}-\boldsymbol{b}-\mathrm{PMLABe}_{\mathbf{7 3}}$}

${ }^{1} \mathrm{H}$ NMR (400.1 MHz; $\left.\left(\mathrm{CD}_{3}\right)_{2} \mathrm{CO}\right), \delta$ (ppm): 2.77-2.83 $(m, 2 \mathrm{nH}$, $\left.\mathrm{CO}_{2} \mathrm{CH}_{2} \mathrm{C}_{6} \mathrm{H}_{5}\right), 3.56-3.59\left(m, 4 \mathrm{mH}(m=42), \mathrm{CH}_{3} \mathrm{O}\left(\mathrm{CH}_{2} \mathrm{CH}_{2} \mathrm{O}\right)_{42}\right)$, 4.97-5.06 ( $\left.m, 2 \mathrm{nH}, \mathrm{CHCH}_{2} \mathrm{CO}_{2}\right), 5.39-5.44\left(m, 1 \mathrm{nH}, \mathrm{CHCH}_{2} \mathrm{CO}_{2}\right)$, 7.19-7.27 ( $\left.m, 5 \mathrm{nH}, \mathrm{CO}_{2} \mathrm{CH}_{2} \mathrm{C}_{6} \mathrm{H}_{5}\right) . \mathrm{M}_{\mathrm{NMR}}=15860 \mathrm{~g} / \mathrm{mol}$ for the PMLABe block. SEC (THF, polystyrene standards, $1 \mathrm{~mL} / \mathrm{min}$ ): $\mathrm{Mw}=5430 \mathrm{~g} / \mathrm{mol}, \mathrm{Ip}=1.38$.

\section{Biot-PEG ${ }_{66}-b-P M L A B e_{73}$}

${ }^{1} \mathrm{H}$ NMR (400.1 MHz; $\left.\left(\mathrm{CD}_{3}\right)_{2} \mathrm{CO}\right), \delta(\mathrm{ppm}): 2.83(s, 2 \mathrm{nH}$, $\left.\mathrm{CO}_{2} \mathrm{CH}_{2} \mathrm{C}_{6} \mathrm{H}_{5}\right)$, 3.56-3.59 ( $m, 4 \mathrm{mH}(m=66)$, $\left.\operatorname{Biot}\left(\mathrm{CH}_{2} \mathrm{CH}_{2} \mathrm{O}\right)_{62}\right)$, 5.00-5.05( $\left.m, 2 \mathrm{nH}, \mathrm{CHCH}_{2} \mathrm{CO}_{2}\right), 5.41-5.44\left(m, 1 \mathrm{nH}, \mathrm{CHCH}_{2} \mathrm{CO}_{2}\right)$, 7.19-7.23 ( $\left.m, 5 \mathrm{nH}, \mathrm{CO}_{2} \mathrm{CH}_{2} \mathrm{C}_{6} \mathrm{H}_{5}\right) . \mathrm{M}_{\mathrm{NMR}}=14630 \mathrm{~g} / \mathrm{mol}$ for the PMLABe block. SEC (THF, polystyrene standards, $1 \mathrm{~mL} / \mathrm{min}$ ): $\mathrm{Mw}=5510 \mathrm{~g} / \mathrm{mol}, \mathrm{Ip}=1.45$.

\section{Preparation of the NPs}

Nanoparticles were prepared by the nanoprecipitation technique described previously (14). Briefly, a solution of $5 \mathrm{mg}$ of copolymer in acetone was rapidly added to $2 \mathrm{~mL}$ of water under vigorous stirring. After $10 \mathrm{~min}$ of stirring at room temperature, acetone was eliminated under vacuum.

A slightly modified nanoprecipitation procedure (Gel filtration method) was also used: $5 \mathrm{mg}$ of block copolymer were dissolved in $100 \mu \mathrm{L}$ of DMF or acetone and $50 \mu \mathrm{L}$ of DMF or acetone, containing or not the $\left.{ }^{185 / 187} \mathrm{Re}\left[\mathrm{PhCS}_{3}\right)_{2}\left(\mathrm{PhCS}_{2}\right)\right]$ ( $\left.{ }^{185 / 187} \mathrm{Re}-\mathrm{SSS}\right)$ prepared as previously described (40), were added. The DMF or acetone solution was rapidly added into $1 \mathrm{~mL}$ of water under vigorous stirring. This solution was stirred at room temperature for $1 \mathrm{~h}$, passed through a gel filtration PD-10 Sephadex G-25 Medium column (GE Healthcare, Little Chalfont, UK) and eluted with $1.5 \mathrm{~mL}+3.5 \mathrm{~mL}$ of water.

\section{Characterization of the Nanoparticles}

The size (average Dh, polydispersity $(Đ)$ and zeta potential of the formulations were measured by respectively DLS and zetametry, without any dilution of the samples.

Nanoparticles were observed by TEM. NPs' suspensions in water were placed on a formvar-carbon film coated on a copper grid (300 mesh). After 6 min of deposition at room temperature, non-adherent NPs were eliminated and the sample was stained by phosphotungstic acid $(0.1 \%)$ for $30 \mathrm{~s}$. TEM imaging was performed under $80 \mathrm{kV}$ accelerating voltage.

\section{Synthesis of ${ }^{99 \mathrm{~m} T C-S S S ~ C o m p l e x}$}

${ }^{99 \mathrm{~m} T c-S S S}$ complex preparation was adapted from previously described method (44). Briefly, kits containing sodium gluconate and tin (II) chloride were first prepared as described thereafter: $825 \mathrm{mg}$ of sodium gluconate were dissolved in $9.9 \mathrm{~mL}$ of distilled water. This solution $(0.9 \mathrm{~mL})$ was placed into 10 flasks. In parallel, $8.25 \mathrm{mg}$ of tin chloride $\left(\mathrm{SnCl}_{2} .2 \mathrm{H}_{2} \mathrm{O}\right)$ were dissolved into $1.1 \mathrm{~mL}$ of $0.1 \mathrm{~N} \mathrm{HCl}$ solution. Then, $0.1 \mathrm{~mL}$ of this solution was added in each flask containing the gluconate solution. The flasks were lyophilized and crimped.

One milliliter of $\left[{ }^{99 \mathrm{~m}} \mathrm{Tc}\right]$-pertechnetate $(750-800 \mathrm{MBq})$, eluted from a ${ }^{99} \mathrm{Mo} /{ }^{99 \mathrm{~m}} \mathrm{Tc}$ generator (ELU III, IBA Molecular, Gif-sur-Yvette, France) was added to a lyophilized kit containing sodium gluconate $(75 \mathrm{mg})$ and $\mathrm{SnCl}_{2}, 2 \mathrm{H}_{2} \mathrm{O}(0.75 \mathrm{mg})$ prepared as described above. The mixture was shaken at room temperature for $15 \mathrm{~min}$. Sodium dithiobenzoate $(10 \mathrm{mg}$ dissolved into $0.5 \mathrm{~mL}$ of $0.9 \%$ saline solution) was added, and the solution was heated at $100^{\circ} \mathrm{C}$ for $15 \mathrm{~min}$. A thin layer chromatography (TLC) was realized in a mixture of hexane and dichloromethane $6 / 4$ as eluent to assess RCP of the complex $\left(\mathrm{R}_{\mathrm{f}}=0.62\right)$. 


\section{Radiolabeling of the NPs}

The ${ }^{99 \mathrm{~m} T c-S S S}$ complex previously prepared was extracted with $1 \mathrm{~mL}$ of dichloromethane. The organic solvent was evaporated at $40^{\circ} \mathrm{C}$. Two procedures were tested for radiolabeling of the NPs according to the ones tested for empty NPs and ${ }^{185 / 187} \mathrm{Re}$-SSS loaded NPs.

\section{Standard Nanoprecipitation}

Acetone or ethanol $(0.5 \mathrm{~mL})$ was then added to the residue to solubilize the complex, and a solution of $5 \mathrm{mg}$ of the selected block copolymers, into $0.5 \mathrm{~mL}$ of acetone, was added. The mixture was nanoprecipitated into $2 \mathrm{~mL}$ of $0.9 \%$ saline solution. After stirring, the organic solvent was eliminated by heating at $45^{\circ} \mathrm{C}$, under slight vacuum, for $15 \mathrm{~min}$. The flask was then centrifuged at 3,500 G for $20 \mathrm{~min}$ (Awel MF 20-R, Blain, France). The supernatant was discarded and the pellet containing the radiolabeled NPs was resuspended into $2 \mathrm{~mL}$ of physiological serum. The radioactivity of the flask before and after centrifugation and resuspension was measured and the encapsulation efficiency was calculated as the ratio of the resuspended pellet activity on total activity before centrifugation.

\section{Gel Filtration}

DMF or acetone $(0.15 \mathrm{~mL})$ was then added to the dried complex. Five milligrams of the selected block copolymer were dissolved into $100 \mu \mathrm{L}$ of DMF or acetone and $50 \mu \mathrm{L}$ of the solution containing the ${ }^{99 \mathrm{~m}} \mathrm{Tc}-\mathrm{SSS}$ complex were added. This organic solution was then rapidly added into $1 \mathrm{~mL}$ of water. The mixture was stirred at room temperature for $15 \mathrm{~min}$, passed through a PD-10 Sephadex G-25 Medium column and eluted with $1.5 \mathrm{~mL}+3.5 \mathrm{~mL}$ of water as described above. The radioactivity of the flask before and after passage on the column was measured and the encapsulation efficiency was calculated as the ratio of the collected fraction's activity on total activity before filtration.

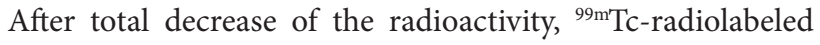
NPs were analyzed by DLS to determine their diameter, polydispersity index, and zeta potential. The radiolabeled NPs were also observed by TEM after complete radioactive decay following the procedure described just above. An energy dispersive X-ray spectrometry (EDS) analysis of the radiolabeled NPs was also realized during the TEM measurements.

\section{Results and Discussion}

We have been recently investigating the possibilities of radiolabeling PMLA derivatives-based NPs with the final goal of using such nanocarriers for the dual site-specific carrying of a radioelement $\left({ }^{99 \mathrm{~m} T c} /{ }^{188} \mathrm{Re}-\mathrm{SSS}\right)$ and an anti-cancer drug. To reach this objective, we studied the possibilities to use degradable stealth and/or targeted NPs, constituted by PMLA amphiphilic derivatives, for radionuclides' encapsulation.

In a first step, we synthesized two amphiphilic PMLA derivatives, $\mathrm{PEG}_{42}-b-\mathrm{PMLABe}_{73}$ and Biot-PEG $66-b$-PMLABe ${ }_{73}$, by anionic ring opening polymerization of the MLABe with, respectively, $\alpha$-methoxy, $\omega$-carboxylate $\mathrm{PEG}_{42}$ and $\alpha$-biotin, $\omega$ carboxylate $\mathrm{PEG}_{66}$ as initiator. MLABe was synthesized in four steps, starting from aspartic acid, following a well-described and reproducible synthetic route (Figure 1) (14-16, 43). After purification by precipitation, allowing the elimination of unreacted initiator and low molecular weight oligomers, block copolymers were characterized by ${ }^{1} \mathrm{H}$ NMR (structure and molecular weight of the PMLABe block) and by SEC (average molecular weight and polymolecularity).

Polymers' characteristics are summarized in Table 1. For both block copolymers, the molecular weights of the PMLABe block were calculated from their ${ }^{1} \mathrm{H}$ NMR spectra on the basis of the integration of peaks corresponding to the PEG block, whose molecular weight is given by the supplier, and on the integration of peaks corresponding to the PMLABe block. Calculated molecular weights were very similar to theoretical ones chosen at $15,000 \mathrm{~g} / \mathrm{mol}$ and fixed by the monomer/initiator ratio. On the other hand, the average molecular weights measured by SEC were lower than the block copolymers' global molecular weights, calculated from the PEG and PMLABe molecular weights, resulting from the fact that THF is not the best solvent for the block copolymers and that the measured molecular

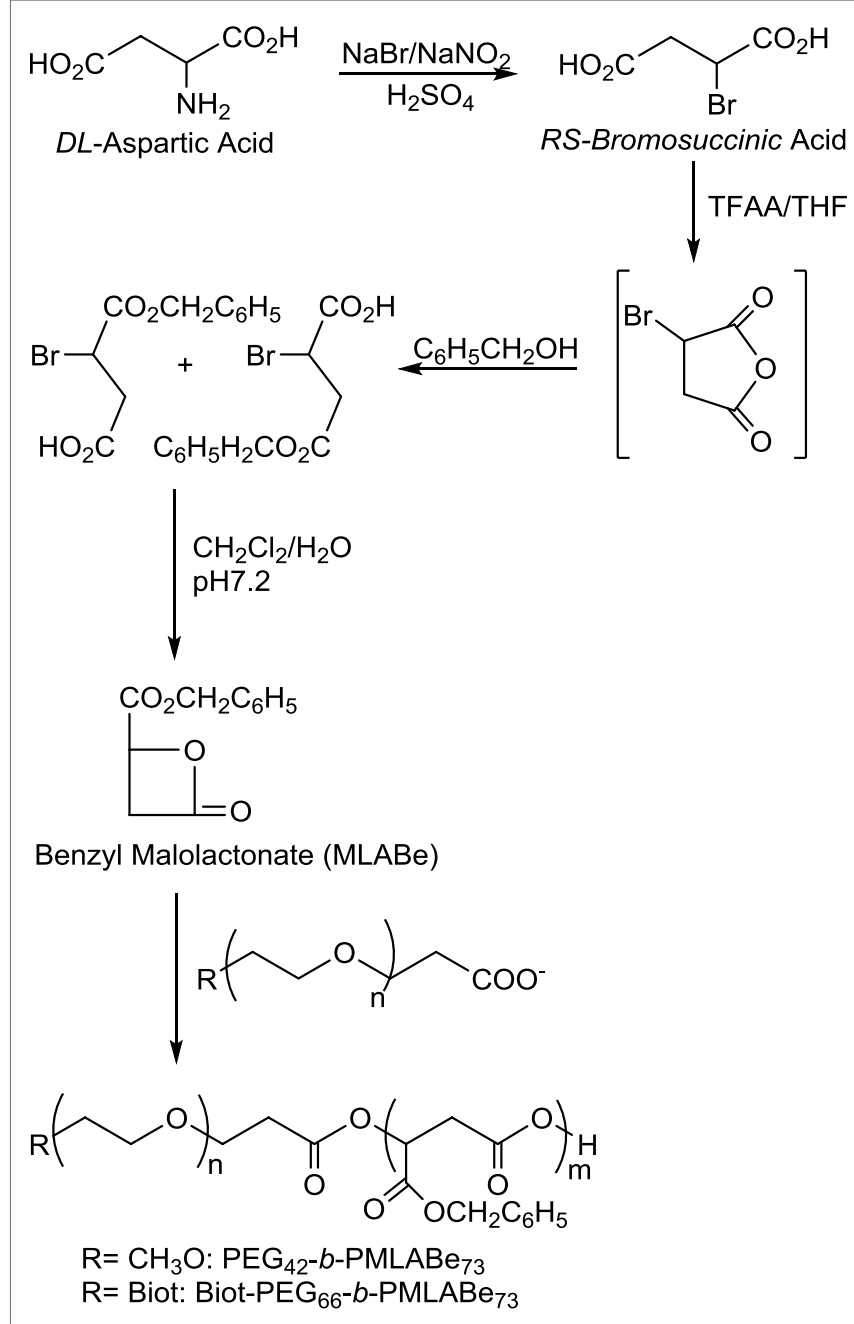

FIGURE 1 | Synthetic route to $\mathrm{PEG}_{42}-b-\mathrm{PMLABe}_{73}$ and Biot-PEG ${ }_{66}-b-$ $\mathrm{PMLABe}_{73}$. 
TABLE 1 | Characteristics of the synthesized block-copolymers.

\begin{tabular}{lcccc}
\hline Block copolymers & $\begin{array}{c}\mathbf{M}_{\mathbf{P E G}}{ }^{a} \\
\mathbf{g} / \mathbf{m o l}\end{array}$ & $\begin{array}{c}\mathbf{M}_{\mathbf{P M L A B e}}{ }^{\mathbf{b}} \\
\mathbf{g} / \mathbf{m o l}\end{array}$ & $\begin{array}{c}\mathbf{M w}^{\mathbf{c}} \\
\mathbf{g} / \mathbf{m o l}\end{array}$ & $\mathbf{I p}^{\mathbf{c}}$ \\
\hline PEG $_{42}-b-\mathrm{PMLABe}_{73}$ & 2015 & 15860 & 5430 & 1.38 \\
Biot-PEG$_{66}-b-\mathrm{PMLABe}$ & 3274 & 14630 & 5510 & 1.45
\end{tabular}

${ }^{a}$ Given by the supplier (PEG IrisBiotech).

${ }^{b}$ Determined by $1 \mathrm{H} N \mathrm{NMR}$ using the relative intensity of protons of PEG and PMLABe blocks.

'Measured by size exclusion chromatography (SEC) in THF at $40^{\circ} \mathrm{C}$ with poly(styrene) standards (1 $\mathrm{mL} / \mathrm{min}, \mathrm{Rl}$ detector).

weights are related to polystyrene standards having a different macromolecular structure than the one of PMLA derivatives. However, SEC analysis allows determining the molecular weight distribution ( $\mathrm{Ip}=\mathrm{Mw} / \mathrm{Mn})$ and verifying the obtaining of a block copolymer (only one peak). In our study, SEC chromatograms of both block-copolymers highlighted the presence of only one macromolecular species (only one peak) and a good control of the polymerization reaction, as shown by the quite low polymolecularity of the samples (Ip $<1.5$ ) in light of these results, we were able to obtain well-defined block copolymers suitable to continue the study of NPs' preparation. However, we still have to further characterize the block copolymers, especially to determine their thermal properties ( $\mathrm{Tg}, \mathrm{Tm}$ ) by differential scanning calorimetry (DSC), which gives an insight on the copolymer state (rubber or glassy state). Nevertheless, we can have a primary idea of the block copolymer's thermal properties since it should have both the thermal properties of each block, i.e., the ones of PEG $\left(\mathrm{Tg}=-20^{\circ} \mathrm{C}, \mathrm{Tm}=50^{\circ} \mathrm{C}\right)$ and the ones of PMLABe $\left(\mathrm{Tg}=37^{\circ} \mathrm{C}\right)$.

Nanoparticles can be easily and reproducibly prepared from these amphiphilic block copolymers by the nanoprecipitation technique, consisting in a rapid addition of a water miscible solvent, in which the block copolymer was dissolved, to an aqueous solution under vigorous stirring at $20^{\circ} \mathrm{C}(14-16)$.

For the standard method, acetone was eliminated under vacuum, while for the gel filtration method, empty NPs and ${ }^{185 / 187}$ Re-SSS loaded NPs were passed through a PD-10 Sephadex G-25 Medium column. The prepared NPs were analyzed by: (i) DLS, giving access to the average $\mathrm{Dh}$ and polydispersity $(\bigoplus)$, (ii) Zetametry, giving access to the surface charge of the NPs, and by (iii) TEM, allowing determining the NPs' morphology.

As shown by results gathered in Table 2, both the standard nanoprecipitation method with acetone and the gel filtration method (with DMF) allowed preparing both stealth and targeted NPs with Dhs varying between 65 and $99 \mathrm{~nm}$ with a quite small size distribution ( $Đ$ lower than 0.15 ), highlighting the formation of well-defined nano-objects in good agreement with the size of micelle-liked NPs (45). On the other hand, the gel filtration method with acetone led to larger NPs with a higher dispersity ( $Đ$ around 0.2). However, the values stayed acceptable. The radiolabeling procedure was first tested with the non-radioactive ${ }^{185 / 187} \mathrm{Re}-\mathrm{SSS}$ complex. The preparation of ${ }^{185 / 187} \mathrm{Re}$-SSS loaded NPs by the standard nanoprecipitation method with acetone led to the formation of large aggregates with quite large size distribution (data not shown) and was not further used in this study. Therefore, we used the gel filtration method toload the ${ }^{185 / 187} \mathrm{Re}-\mathrm{SSS}$
TABLE 2 | Characteristics of empty and ${ }^{185 / 187}$ Re-SSS loaded PMLABe derivatives-based NPs.

\begin{tabular}{|c|c|c|c|}
\hline NPs & 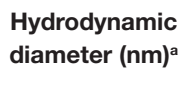 & $\begin{array}{l}\text { Polydispersity } \\
\text { index }(\bigoplus)^{\mathrm{a}}\end{array}$ & $\begin{array}{c}\text { Zeta } \\
\text { potential } \\
(\mathrm{mV})^{\mathrm{b}}\end{array}$ \\
\hline \multicolumn{4}{|l|}{$\mathrm{PEG}_{42}-b-\mathrm{PMLABe} \mathrm{e}_{73}$} \\
\hline $\begin{array}{l}\text { Standard nanoprecipitation } \\
\text { method with acetone }\end{array}$ & 80 & 0.06 & -6 \\
\hline Gel filtration with acetone & 113 & 0.17 & -31 \\
\hline Gel filtration with DMF & 99 & 0.11 & -29 \\
\hline \multicolumn{4}{|l|}{ Biot-PEG ${ }_{66}-b-P M L A B e_{73}$} \\
\hline $\begin{array}{l}\text { Standard nanoprecipitation } \\
\text { method with acetone }\end{array}$ & 65 & 0.12 & -7 \\
\hline $\begin{array}{l}\text { Standard radiolabeling } \\
\text { method with acetone }\end{array}$ & 80 & 0.21 & - \\
\hline Gel filtration with acetone & 87 & 0.20 & -26 \\
\hline Gel filtration with DMF & 68 & 0.15 & -30 \\
\hline \multicolumn{4}{|c|}{$\mathrm{PEG}_{42}-b-\mathrm{PMLABe} \mathrm{e}_{73}\left[{ }^{185 / 187} \mathrm{Re}-\mathrm{SSS}\right]$} \\
\hline Gel filtration with acetone & 174 & 0.17 & -37 \\
\hline Gel filtration with DMF & 104 & 0.14 & -32 \\
\hline \multicolumn{4}{|c|}{ Biot-PEG $66-b-P M L A B e_{73}\left[{ }^{185 / 187} R e-S S S\right]$} \\
\hline Gel filtration with acetone & 135 & 0.18 & -25 \\
\hline Gel filtration with DMF & 99 & 0.15 & -39 \\
\hline
\end{tabular}

a Measured by dynamic light scattering (DLS).

${ }^{b}$ Measured by zetametry.

complex inside the NPs. As shown by results given in Table 2, gel filtration with DMF allowed obtaining quite well-defined NPs (low $Đ$ ) with a slightly increased Dh, probably as a result of the presence of the complex inside de NPs. On the contrary, with acetone, it led to larger NPs with higher polydispersity indexes. At this stage, we can conclude that gel filtration with DMF seems to lead to the best results in terms of size and size distribution. Stirring the solution for $15 \mathrm{~min}$, instead of $1 \mathrm{~h}$ leads to not as good results (Dhs and $Đ$, respectively, $144 \mathrm{~nm}$ vs. 104 and 0.19 vs. 0.14 for ${ }^{185 / 187} \mathrm{Re}-\mathrm{SSS}$ loaded $\mathrm{PEG}_{42}-b-\mathrm{PMLABe}_{73}$ in $\mathrm{DMF}$ ), but it is better suited for the encapsulation of technetium-99m, due to radioactive decay considerations, and values are still satisfactory. The NPs prepared by the gel filtration methods (acetone and DMF) loaded or not with the ${ }^{185 / 187} \mathrm{Re}-\mathrm{SSS}$ complex have zeta potentials around $-30 \mathrm{mV}$ highlighting a negative surface charge which can be associated with a good stability of the corresponding NPs. Such negative surface charges can be the result of a block copolymer chain conformation allowing the presence of carboxylate end groups of the PMLABe block at the surface of the NPs thus lowering the values of the zeta potential. However, further experiments (static light scattering, Cryo-TEM, etc.) are needed to determine the morphology of the NPs and if any change can be observed in function of the preparation procedure used to obtain the NPs.

In parallel, the selected radioactive complex $\left[{ }^{99 \mathrm{~m}} \mathrm{Tc}\left(\mathrm{PhCS}_{3}\right)_{2}\left(\mathrm{PhCS}_{2}\right)\right]$ ( $\left.{ }^{99 \mathrm{~m}} \mathrm{Tc}-\mathrm{SSS}\right)$ has been prepared in two steps, starting from the generator-eluted pertechnetate ion,

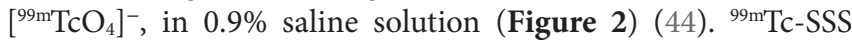
complex was obtained with a RCP over $90 \%$.

After those steps, we had to set up a simple and reproducible way of radiolabeling $\mathrm{PEG}_{42}-b-\mathrm{PMLABe}_{73}$ or Biot- $\mathrm{PEG}_{66}-b-\mathrm{PMLABe}_{73}$ 


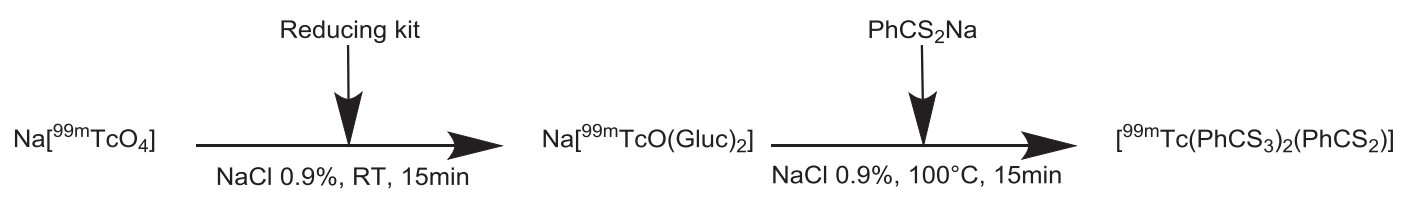

FIGURE 2 | Synthesis of the ${ }^{99 m}$ T-SSS radiocomplex

TABLE 3 | Characteristics of radiolabeled PMLABe derivatives-based NPs.

\begin{tabular}{|c|c|c|c|c|}
\hline NPs & $\begin{array}{l}\text { Encapsulation } \\
\text { efficiency }(\%)^{a}\end{array}$ & $\begin{array}{l}\text { Hydrodynamic } \\
\text { diameter }(\mathrm{nm})^{\mathrm{b}}\end{array}$ & $\begin{array}{l}\text { Polydispersity } \\
\text { index }(\Xi)^{\mathrm{b}}\end{array}$ & $\begin{array}{c}\text { Zeta potential } \\
(\mathrm{mV})^{\mathrm{b}}\end{array}$ \\
\hline \multicolumn{5}{|l|}{$\mathrm{PEG}_{42}-b-\mathrm{PMLABe}_{73}\left[{ }^{99 \mathrm{~m}} \mathrm{Tc}-\mathrm{SSS}\right]$} \\
\hline Standard nanoprecipitation method with acetone & 61 & 230 & 0.15 & +0.2 \\
\hline Gel filtration with DMF & 86 & 103 & 0.16 & -38 \\
\hline \multicolumn{5}{|l|}{ Biot-PEG 6 6-b-PMLABe ${ }_{73}\left[{ }^{99 m} \mathrm{Tc}-\mathrm{SSS}\right]$} \\
\hline Standard nanoprecipitation method with acetone & 82 & 160 & 0.23 & -4.3 \\
\hline Gel filtration with DMF & 89 & 249 & 0.22 & -25 \\
\hline
\end{tabular}

${ }^{a}$ Determined by radioactivity measurement.

${ }^{b}$ Measured by DLS (Delsa Nano ${ }^{\circledR}$ Beckman Coulter).

based NPs. In a first attempt, we tried to encapsulate the ${ }^{99 \mathrm{~m} T c-S S S}$ complex in already formed NPs, previously obtained by the nanoprecipitation technique as described above. However, we were not able to encapsulate the complex inside the preformed NPs because of their destabilization by the reducing agent which is present in the kits used for the complex formation. Consequently, it was necessary to extract the complex in dichloromethane in order to eliminate the kit constituents. Dichloromethane was then evaporated and the radiotracer retaken in a water-miscible organic solvent, then added to the acetone or DMF solution containing $\mathrm{PEG}_{42}-b-\mathrm{PMLABe}_{73}$ or Biot-PEG $\mathrm{PL}_{66}-b-\mathrm{PMLABe}_{73}$ block copolymers.

To determine the encapsulation efficiency, we have measured the NPs' radioactivity. For that, we have eliminated the nonencapsulated complex:

- $\quad$ either by centrifugation in the case of the standard nanoprecipitation using acetone: the supernatant was discarded and the pellet was again suspended into physiological serum. The total radioactivity of the flask before and after centrifugation and the radioactivity of the supernatant were measured. The encapsulation efficiency was given by the ratio between the radioactivity of the flask before centrifugation and the radioactivity of the flask after centrifugation and resuspension of NPs (Table 3). Some trials have been done with ethanol to solubilize ${ }^{99 \mathrm{~m}} \mathrm{Tc}$-SSS after dichloromethane evaporation, but this solvent is more difficult to evaporate than acetone, so the latter one was preferred.

- $\quad$ or by gel filtration through a PD-10 Sephadex G-25 Medium column. The total radioactivity of the flask before and after Sephadex filtration were measured and allowed to calculate the encapsulation efficiency (ratio between the radioactivity of the flask before and after filtration) (Table 3).
After the radioactivity decay, Dhs, polydispersity indexes, and zeta potentials of the complex-containing NPs were measured by DLS. As shown by results gathered in Table 3, ${ }^{99 \mathrm{~m}} \mathrm{Tc}-\mathrm{SSS}$ complex was successfully encapsulated in both $\mathrm{PEG}_{42}-b-\mathrm{PMLABe}_{73}$ and Biot-PEG ${ }_{66}-b-\mathrm{PMLABe}_{73}$ based NPs, using standard nanoprecipitation technique, with an encapsulation efficiency varying between 60 and 80\%. Non-PEGylated PMLABe based NPs were also investigated to encapsulate ${ }^{99 m} \mathrm{Tc}-S S S$, but the resulting labeled NPs were not sufficiently stable in time to be studied after radioactivity decay. The radiolabeled NPs, obtained through the standard labeling method, present increased diameters with identical low polydispersity indexes, typically in the $0.15-0.23$ range, and neutral zeta potentials in comparison to non-radiolabeled NPs (Table 3). Several reasons can explain the increase of the diameters measured by DLS: (i) a swelling of the inner-core by remaining acetone which is quite difficult to totally eliminate in the experimental conditions of the standard nanoprecipitation procedure used to prepare radiolabeled NPs, (ii) the presence of the radiotracer itself, or (iii) an aggregation of the NPs during the time necessary for radioactivity decay (3 days). Except for the simple PMLABe NPs, the NPs demonstrated good in vitro stability, with no size change over time (Table 4). Thus, the increase in size cannot be explained by the delay for the radioactivity to decay before measurements. The results obtained on empty NPs prepared using the same protocol than the one used for the preparation of radiolabeled NPs (standard radiolabeling method), and with NPs encapsulating non-radioactive complex based on "cold" ${ }^{185 / 187}$ Re evidenced that the presence of the complex leads to an increase of the NPs' diameters without aggregation (Table 2). However, because the total elimination of acetone is quite difficult with the radioactive complex and centrifugation procedure is not satisfactory, with NPs still present in the supernatant, as evidenced by DLS measurements, thus 
TABLE 4 | Over-time stability of PMLABe derivatives-based NPs (measured by DLS).

\begin{tabular}{|c|c|c|}
\hline NPs & $\begin{array}{l}\text { Hydrodynamic } \\
\text { diameter (nm) }\end{array}$ & $\begin{array}{c}\text { Polydispersity } \\
\text { index (Đ) }\end{array}$ \\
\hline \multicolumn{3}{|c|}{$\mathrm{PEG}_{42}-b-\mathrm{PMLABe} \mathrm{e}_{73}$} \\
\hline D0 (acetone) & 113 & 0.17 \\
\hline D7 (acetone) & 119 & 0.13 \\
\hline DO (DMF) & 99 & 0.11 \\
\hline D7 (DMF) & 105 & 0.10 \\
\hline \multicolumn{3}{|c|}{ Biot-PEG ${ }_{66}-b-P M L A B e_{73}$} \\
\hline D0 (acetone) & 87 & 0.20 \\
\hline D7 (acetone) & 87 & 0.19 \\
\hline DO (DMF) & 68 & 0.15 \\
\hline D7 (DMF) & 88 & 0.15 \\
\hline
\end{tabular}

probably minoring encapsulation yields, we tried to purify the radiolabeled NPs by dialysis. However, such method was time consuming, which is crippling with short half-life radionuclides, and led to no better results than centrifugation, so this method was not pursued.

We therefore used the gel filtration method to prepare radiolabeled NPs. Using this gel filtration procedure, we also observed larger Dhs with slightly higher polydispersity index values compared to empty NPs (Table 3). This increase in diameters is probably the result of the radiotracer encapsulation, because no significant differences were observed in diameter and polydispersity index values between the standard nanoprecipitation procedure and the gel filtration method (Table 2). However, the encapsulation efficiencies seem to be slightly higher when gel filtration method is used either with acetone or DMF, with encapsulation yields between 86 and $89 \%$. Moreover, this procedure is faster and easier to set up than the standard nanoprecipitation method because no centrifugation is necessary, thus avoiding the difficulties to separate the supernatant and the pellet. As observed previously with empty NPs, this method led to NPs with negative zeta potentials despite the PEG chains. As already mentioned, further experiments will be done to understand the reasons for this observed phenomenon which is probably related to the chain conformation adopted by the block copolymer as mentioned above.

Both empty and radiolabeled NPs, after radioactivity decay, were analyzed by TEM in order to have an idea of their morphology (Figure 3).

Indeed, DLS allows only measuring Dh and size distribution without having access to NPs' morphology. As shown by Figure 3, both empty and radiolabeled NPs, prepared with the standard method, have a spherical shape with a diameter in good agreement with the Dhs measured by DLS: around $80 \mathrm{~nm}$ for empty NPs and $120 \mathrm{~nm}$ for radiolabeled NPs. Moreover, an EDS analysis of the radiolabeled NPs was also realized during the TEM measurements: this technique allows the determination of the elements present in the analyzed sample (46). EDS analysis evidenced the presence of sulfur inside the NPs, reflecting the presence of the ${ }^{99 \mathrm{~m} T c-S S S}$ complex which contains sulfur atoms. On the contrary, EDS analysis realized on empty NPs did not highlight any presence of sulfur atoms. Therefore, it tends to
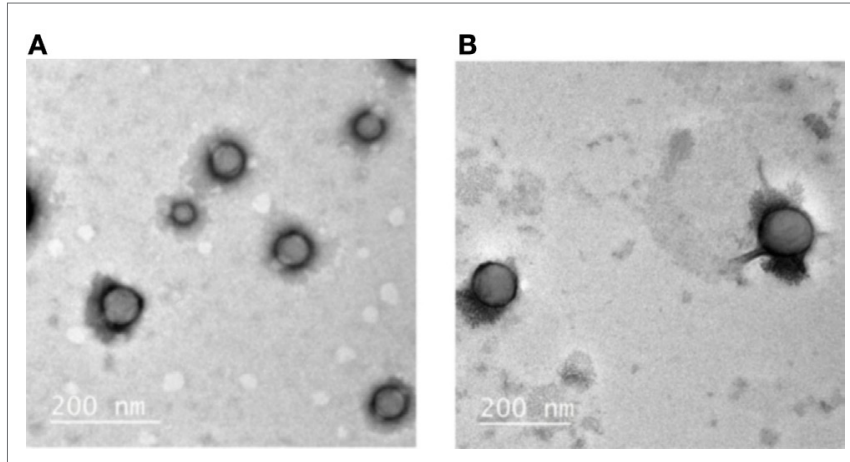

FIGURE 3 | TEM images of (A) Empty Biot-PEG ${ }_{66}-b-$ PMLABe $_{73}$ based NPs and (B) Radiolabeled Biot-PEG ${ }_{66}-b-\mathrm{PMLABe}_{73}$ based NPs.

prove that the ${ }^{99 \mathrm{~m}} \mathrm{Tc}-\mathrm{SSS}$ complex is effectively encapsulated inside the NPs constituting by PMLA derivatives. TEM experiments now need to be done on NPs prepared using the alternative gel filtration method to get more insight on the influence of the preparation method on NPs morphology.

\section{Conclusion}

Through this study, we highlighted that it is possible to obtain radiolabeled NPs based on PEGylated or biotinylated PEGylated degradable block copolymers with quite high encapsulation efficiency (up to $89 \%$ ) in an easy and reproducible way. The radiolabeled NPs were characterized by Dhs ranging from 100 to $230 \mathrm{~nm}$ with quite low polydispersity indexes (under 0.2 ) and negative zeta potentials. The morphology of such NPs was shown to be spherical and the presence of sulfur, resulting from ${ }^{99 \mathrm{~m}} \mathrm{Tc}$ SSS complex, was detected inside the NPs.

We are now moving to ${ }^{188} \mathrm{Re}$-SSS encapsulation and in vitro assays to confirm the potential of stealth and targeting NPs based on PMLABe derivatives as therapeutic nanovectors. Since our team has been working on DOX's encapsulation and the grafting of a fluorescent probe too, we likewise envision to combine these different approaches as potential theranostic tools of interest for combined chemo-/radiotherapy and/or bimodal imaging probes for instance.

\section{Author Contributions}

$\mathrm{NL}$ and SC-M designed and supervised the project, participated in the experiments and the analysis and interpretation of data, and wrote the manuscript. $\mathrm{LC}, \mathrm{MB}, \mathrm{CB}, \mathrm{CR}$, and $\mathrm{MD}$ made contribution in the design of the work, realized the experiments, and analyzed the results. NN designed and supervised the project and participated in the interpretation of data.

\section{Acknowledgments}

Vincent Dorcet and Thomas Vives are gratefully acknowledged for their help for, respectively, TEM and DLS measurements. This work was supported by Labex IRON (Grant no. ANR-11-LABX-0018). 


\section{References}

1. Wicki A, Witzigmann D, Balasubramanian V, Huwyler J. Nanomedicine in cancer therapy: challenges, opportunities, and clinical applications. J Control Release (2015) 200:138-57. doi:10.1016/j.jconrel.2014.12.030

2. Reddy LH, Couvreur P. Nanotechnology for therapy and imaging of liver diseases. J Hepatol (2011) 55:1461-6. doi:10.1016/j.jhep.2011.05.039

3. Elsaesser A, Howard CV. Toxicology of nanoparticles. Adv Drug Deliv Rev (2012) 64:129-37. doi:10.1016/j.addr.2011.09.001

4. Yan Y, Such GK, Johnston APR, Best JP, Caruso F. Engineering particles for therapeutic delivery: prospects and challenges. ACS Nano (2012) 6:3663-9. doi:10.1021/nn3016162

5. Malam Y, Loizidou M, Seifalian AM. Liposomes and nanoparticles: nano-sized vehicles for drug delivery in cancer. Trends Pharmacol Sci (2009) 30:592-9. doi:10.1016/j.tips.2009.08.004

6. Misra R, Acharya S, Sahoo SK. Cancer nanotechnology: application of nano- technology in cancer therapy. Drug Discov Today (2010) 15:842-50. doi:10.1016/j.drudis.2010.08.006

7. Chiellini F, Piras AM, Errico C, Chiellini E. Micro/nanostructured polymeric systems for biomedical and pharmaceutical applications. Nanomedicine (2008) 3:367-93. doi:10.2217/17435889.3.3.367

8. Kumari A, Yadav SK, Yadav SC. Biodegradable polymeric nanoparticles based drug delivery systems. Colloids Surf B Biointerfaces (2010) 75:1-18. doi:10.1016/j.colsurfb.2009.09.001

9. Piras AM, Chiellini F, Fiumi C, Bartoli C, Chiellini E, Fiorentino B, et al. New biocompatible nanoparticle delivery system for the release of fibrinolytic drugs. Int J Pharm (2008) 357:260-71. doi:10.1016/j. ijpharm.2008.01.035

10. Dessy A, Piras AM, Dinucci D, Chiellini F. 2-methoxy aniline grafted poly(maleic anhydride- alt- butyl vinyl ether) hemiester: a NEW biocompatible polymeric free radical scavenger. Macromolecules (2011) 44:848-56. doi:10.1021/ ma102309m

11. Vert M, Lenz RW. Preparation and properties of poly- $\beta$-malic acid: a functional polyester of potential biomedical importance. Polym Preprints (1979) 20:608-11.

12. Cammas S, Béar MM, Moine L, Escalup R, Ponchel G, Kataoka K, et al. Polymers of malic acid and 3-alkylmalic acid as synthetical PHAs in the design of biocompatible hydrolyzable devices. Int J Biol Macromol (1999) 25:273-82. doi:10.1016/S0141-8130(99)00042-2

13. Cammas-Marion S. Guerin Ph design of malolactonic acid esters with a large spectrum of specified pendant groups in the engineering of biofunctional and hydrolyzable polyesters. Macromol Symp (2000) 153:167-86. doi:10.1002/1521-3900(200003)153:1<167::AID-MASY167>3.0.CO;2-Q

14. Huang ZW, Laurent V, Chetouani G, Ljubimova JY, Holler E, Benvegnu T, et al. New functional degradable and bio-compatible nanoparticles based on poly(malic acid) derivatives for site-specific anticancer drug delivery. Int $J$ Pharm (2012) 423:84-92. doi:10.1016/j.ijpharm.2011.04.035

15. Loyer P, Bedhouche W, Huang ZW, Cammas-Marion S. Degradable and biocompatible nanoparticles decorated with cyclic RGD peptide for efficient drug delivery to hepatoma cells in vitro. Int J Pharm (2013) 454:727-37. doi:10.1016/j.ijpharm.2013.05.060

16. Cammas-Marion S, Loyer P. Natural and synthetic poly(malic acid)based derivates: a family of versatile biopolymers for the design of drug nanocarriers. J Drug Target (2014) 22:556-75. doi:10.3109/10611 86X.2014.936871

17. Ting G, Chang $\mathrm{CH}$, Wang HE. Cancer nanotargeted radiopharmaceuticals for tumor imaging and therapy. Anticancer Res (2009) 29:4107-18.

18. Muthu MS, Wilson B. Multifunctional radionanomedicine: a novel nanoplatform for cancer imaging and therapy. Nanomedicine (2010) 5:169-71. doi:10.2217/nnm.09.107

19. Huang WY, Davis JJ. Multimodality and nanoparticles in medical imaging. Dalton Trans (2011) 40:6087-103. doi:10.1039/c0dt01656j

20. De Barros ALB, Tsourkas A, Saboury B, Cardoso VN, Alavi A. Emerging role of radiolabeled nanoparticles as an effective diagnostic technique. EJNMMI Res (2012) 2:39-53. doi:10.1186/2191-219X-2-39
21. Lee DH, Koo H, Sun IC, Ryu JH, Kim K, Kwon IC. Multifunctional nanoparticles for multimodal imaging and theragnosis. Chem Soc Rev (2012) 41:2656-72. doi:10.1039/C2CS15261D

22. Vanpouille-Box C, Hindré F. Nanovectorized radiotherapy: a new strategy to induce anti-tumor immunity. Front Oncol (2012) 2:136. doi:10.3389/ fonc. 2012.00136

23. Guo Y, Aweda T, Black KCL, Liu Y. Chemistry and theranostic applications of radiolabeled nanoparticles for cardiovascular, oncological, and pulmonary research. Curr Top Med Chem (2013) 13:470-8. doi:10.2174/15680266113130 40007

24. Mitra A, Nan A, Line BR, Ghandehari H. Nanocarriers for nuclear imaging and radiotherapy of cancer. Curr Pharm Des (2006) 12:4729-49. doi:10.2174/138161206779026317

25. Peng CL, Shih YH, Lee PC, Mon-Hsian Hsieh T, Luo TY, Shieh MJ. Multimodal image-guided photothermal therapy mediated by ${ }^{188} \mathrm{Re}$-labeled micelles containing a cyanine-type photosensitizer. ACS Nano (2011) 5:5594-607. doi:10.1021/nn201100m

26. Polyák A, Hajdu I, Bodnár M, Trencsényi G, Pöstényi Z, Haász V, et al. ${ }^{99 \mathrm{~m} T c-l a b e l l e d ~ n a n o s y s t e m ~ a s ~ t u m o u r ~ i m a g i n g ~ a g e n t ~ f o r ~ S P E C T ~ a n d ~}$ SPECT/CT modalities. Int J Pharm (2013) 449:10-7. doi:10.1016/j. ijpharm.2013.03.049

27. Phillips WT, Bao A, Sou K, Li S, Goins B. Radiolabeled liposomes as drug delivery nanotheranostics. In: Li C, Tian M, editors. Drug Delivery Applications of Noninvasive Imaging: Validation from Biodistribution to Sites of Action. Hoboken, NJ: John Wiley \& Sons, Inc (2013). p. 252-67.

28. Allard E, Hindre F, Passirani C, Lemaire L, Lepareur N, Noiret N, et al. ${ }^{188} \mathrm{Re}$-loaded lipid nanocapsules as a promising radiopharmaceutical carrier for internal radiotherapy of malignant gliomas. Eur J Nucl Med Mol Imaging (2008) 35:1838-46. doi:10.1007/s00259-008-0735-Z

29. Kovacs L, Tassano M, Cabrera M, Fernandez M, Porcal W, Anjos RM, et al. Labeling polyamidoamine (PAMAM) dendrimers with technetium-99m via hydrazinonicotinamide (HYNIC). Curr Radiopharm (2014) 7:115-22. doi:10. 2174/1874471007666140825121615

30. Torres Martin de Rosales R, Tavaré R, Glaria A, Varma G, Protti A, Blower PJ. ${ }^{99 \mathrm{~m} T c-b i s p h o s p h o n a t e-i r o n ~ o x i d e ~ n a n o p a r t i c l e ~ c o n j u g a t e s ~ f o r ~ d u a l-m o d a l i t y ~}$ biomedical imaging. Bioconjug Chem (2011) 22:455-65. doi:10.1021/ bc100483k

31. Dams ETM, Oyen WJG, Boerman OC, Storm G, Laverman P, Kok PJM, et al. ${ }^{99 \mathrm{~m}} \mathrm{Tc}-\mathrm{PEG}$ liposomes for the scintigraphic detection of infection and inflammation: clinical evaluation. J NucI Med (2000) 41:622-30.

32. Pereira MA, Mosqueira VCF, Carmo VA, Ferrari CS, Reis ECO, Ramaldes GA, et al. Biodistribution study and identification of inflammatory sites using nanocapsules labeled with ${ }^{99 \mathrm{~m}} \mathrm{Tc}-\mathrm{HMPAO}$. Nucl Med Commun (2009) 30:749-55. doi:10.1097/MNM.0b013e32832f2b59

33. Torres Miranda Sa L, de Souza Albernaz M, Ferreira de Carvalho Patricio B, Villela Falcão Junior M, Feliciano Coelho B, Bordim A, et al. Biodistribution of nanoparticles: initial considerations. J Pharm Biomed Anal (2012) 70:602-4. doi:10.1016/j.jpba.2012.06.008

34. Iyer $\mathrm{AK}, \mathrm{He} \mathrm{J}$, Amiji MM. Image-guided nanosystems for targeted delivery in cancer therapy. Curr Med Chem (2012) 19:3230-40. doi:10.2174/092986712800784685

35. Snehalatha M, Kolachina V, Saha RN, Babbar AK, Sharma N, Sharma RK. Enhanced tumor uptake, biodistribution and pharmacokinetics of etoposide loaded nanoparticles in Dalton's lymphoma tumor bearing mice. J Pharm Bioallied Sci (2013) 5:290-7. doi:10.4103/0975-7406.120081

36. Vanpouille-Box C, Lacoeuille F, Belloche C, Lepareur N, Lemaire L, Le Jeune $\mathrm{JJ}$, et al. Tumor eradication in rat glioma and bypass of immunosuppressive barriers using internal radiation with ${ }^{188}$ Re-lipid nanocapsules. Biomaterials (2011) 32:6781-90. doi:10.1016/j.biomaterials.2011.05.067

37. Douglas SJ, Davis SS. Radiolabelling of poly(butyl 2-cyanoacrylate) nanoparticles with a technetium-99m-dextran complex. J Labelled Comp Radiopharm (1986) 23:495-504. doi:10.1002/jlcr.2580230506

38. Lambert B, de Klerk JMH. Clinical applications of ${ }^{188} \mathrm{Re}$-labelled radiopharmaceuticals for radionuclide therapy. Nucl Med Commun (2006) 27:223-9. doi:10.1097/00006231-200603000-00004 
39. Pillai MRA, Dash A, Knapp FF Jr. Rhenium-188: availability from the ${ }^{188} \mathrm{~W} /{ }^{188} \mathrm{Re}$ generator and status of current applications. Curr Radiopharm (2012) 5:228-43. doi:10.2174/1874471011205030228

40. Lepareur N, Mévellec F, Noiret N, Refosco F, Tisato F, Porchia M, et al. Syntheses and reactivity of " sulphur rich " $\operatorname{Re}($ III) and $\operatorname{Tc}$ (III) complexes containing trithioperoxybenzoate, dithiobenzoate and dithiocarbamate ligands. Dalton Trans (2005) 17:2866-75. doi:10.1039/ b503938j

41. Heeg MJ, Jurisson SS. The role of inorganic chemistry in the development of radiometal agents for cancer therapy. Acc Chem Res (1999) 32:1053-60. doi:10.1021/ar980002c

42. Ballot S, Noiret N, Hindré F, Denizot B, Garin E, Rajerison H, et al. ${ }^{99 \mathrm{~m}} \mathrm{Tc} /{ }^{188} \mathrm{Re}$-labelled lipid nanocapsules as promising radiotracers for imaging and therapy: formulation and biodistribution. Eur J Nucl Med Mol Imaging (2006) 33:602-7. doi:10.1007/s00259-005-0007-0

43. Cammas S, Renard I, Langlois V. Guérin Ph Poly( $\beta$-malic acid): obtaining of high molecular weights by improvement of the synthesis route. Polymer (1996) 37(18):4215-20. doi:10.1016/0032-3861(96)00204-2

44. Garin E, Noiret N, Malbert CH, Lepareur N, Roucoux A, Dazord L, et al. Development of ${ }^{99 \mathrm{~m}} \mathrm{Tc}$ labelled Lipiodol: biodistribution following injection into the hepatic artery of the healthy pig. Nucl Med Commun (2004) 25:291-8. doi:10.1097/00006231-200403000-00013

45. Yokoyama M, Kwon GS, Okano T, Sakurai Y, Seto T, Kataoka K. Preparation of micelle-forming polymer-drug conjugates. Bioconjugate Chem (1992) 3:295-301. doi:10.1021/bc00016a007

46. Keil K, Fitzgerald R, Heinrich KF. Celebrating 40 years of energy dispersive $\mathrm{X}$-ray spectrometry in electron probe microanalysis: a historic and nostalgic look back into the beginnings. Microsc Microanal (2009) 15:476-83. doi:10.1017/S1431927609990377

Conflict of Interest Statement: The authors declare that the research was conducted in the absence of any commercial or financial relationships that could be construed as a potential conflict of interest.

Copyright (C) 2015 Lepareur, Leal E Costa, Bocqué, Blondelle, Ruello, Desjulets, Noiret and Cammas-Marion. This is an open-access article distributed under the terms of the Creative Commons Attribution License (CC BY). The use, distribution or reproduction in other forums is permitted, provided the original author(s) or licensor are credited and that the original publication in this journal is cited, in accordance with accepted academic practice. No use, distribution or reproduction is permitted which does not comply with these terms. 